\title{
Vacinação contra o papiloma vírus humano no género masculino em idade pediátrica: qual a evidência?
}

Vanessa Filipa Guedes Silva, ${ }^{1}$ Diana Soares, ${ }^{2}$ Sofia Rodrigues, Cátia Lírio, Marisa Barros

\section{RESUMO}

Objetivo: Avaliar a eficácia da vacinação contra o papiloma vírus humano (HPV) no género masculino, em idade pediátrica, na prevenção de patologia maligna ou pré-maligna associada a infeção por HPV.

Fontes de dados: PubMed, The Cochrane Library, DARE, National Guideline Clearinghouse, Canadian Medical Association Practice Guidelines InfoBase e Guidelines Finder (National Electronic Library for Health - NHS britânico).

Métodos de revisão: Pesquisa de artigos publicados entre 01/01/2006 e 01/09/2016, usando os termos MeSH Papillomavirus vaccines, male, child e adolescent. Foram incluídos indivíduos do género masculino em idade pediátrica com vacinação contra o HPV para prevenção de patologia oncológica ou pré-oncológica por HPV. Para atribuição do nível de evidência (NE) e a força de recomendação foi utilizada a escala Strengh of Recomendation Taxonomy, da American Academy of Family Physicians.

Resultados: Nas três normas de orientação clínica encontradas existe uma força de recomendação A para a vacinação contra o HPV no género masculino em idade pediátrica. Dos 11 artigos incluídos, a maioria descreve que a vacinação contra o HPV no género masculino em idade pediátrica conduz à redução de incidência de infeção por HPV e lesões malignas e/ou pré-malignas.

Conclusões: A vacinação contra o HPV é eficaz na prevenção de lesões pelo HPV, pelo que está recomendada no género masculino em idade pediátrica (força de recomendação A). A vacinação mista é mais eficaz do que a vacinação isolada do género feminino.

Palavras-chave: Adolescente; Criança; Género masculino; Vacina papilomavírus.

\section{INTRODUÇÃO}

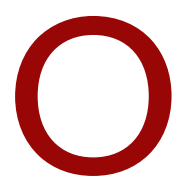

papiloma vírus humano (HPV) é um dos vírus de transmissão sexual mais frequente, provocando infeção do epitélio escamoso. ${ }^{1-2}$ A maioria das infeções por HPV é transitória, tendo duração de alguns meses. Em alguns casos a infeção pode ser persistente, progredindo para lesões pré-oncológicas ou oncológicas. ${ }^{1} \mathrm{O}$ HPV é a maior causa de cancro de colo do útero nas mulheres; no entanto, pode também causar doença no género masculino. As estirpes de alto risco, que são fatores importantes para o cancro cervical, vulvar, vaginal, anal,

1. USF Nova Salus.

2. USF Abel Salazar. peniano e orofaríngeo, incluem o HPV-16, 18 e $45 .^{1,3}$ As estirpes de baixo risco incluem o HPV-6 e 11, sendo maioritariamente responsáveis por condilomas genitais e papilomatose respiratória. ${ }^{1,3}$

Existem três tipos de vacinas para o HPV, a bivalente (HPV 16 e 8), a quadrivalente (HPV 6, 11; 16 e 18) e a nonavalente (HPV 6, 11, 16, 18, 31, 33, 45, 52 e 58). ${ }^{1,3} \mathrm{Em}$ Portugal, de acordo com o Programa Nacional de Vacinação (PNV) de 2017, recomenda-se a realização da vacina HPV nonavalente aos 10 anos no género feminino. ${ }^{4}$ A Áustria foi o primeiro país a implementar um programa nacional de vacinação contra o HPV que abrange o género masculino e feminino. ${ }^{1}$ Existem também recomendações para a vacinação no género masculino na Austrália, Canadá e Estados Unidos da Amé- 
rica. ${ }^{5}$ Contudo, a proteção da vacina HPV parece não ser duradoura. A vacina quadrivalente confere uma proteção média de oito anos..$^{6-8}$

O objetivo do estudo é avaliar a eficácia da vacinação contra o HPV no género masculino, em idade pediátrica, na prevenção de patologia maligna ou pré-maligna associada a infeção por HPV.

\section{MÉTODOS}

Fonte de dados: PubMed, The Cochrane Library, DARE, National Guideline Clearinghouse, Canadian MedicalAssociation Practice Guidelines InfoBase e Guidelines Finder (National Electronic Library for Health - NHS britânico).

\section{Métodos de revisão}

Foi realizada uma pesquisa de normas de orientação clínica (NOC), revisões sistemáticas (RS), meta-análises (MA), ensaios clínicos aleatorizados e controlados (ECAC), com data de publicação entre 1 de janeiro de 2006 e 1 de setembro de 2016, utilizando os termos MeSH Papillomavirus vaccines, male, child e adolescent. Em agosto de 2018 foi realizada uma atualização das guidelines, tendo sido incluídas a Advisory Committee on Immunization Practices (ACIP) de 2016, ${ }^{9}$ do Centers for Disease Control and Prevention (CDC), a $\mathrm{Na}$ tional Advisory Committee on Immunization (NACI) de $2017,{ }^{10}$ do Canadá e a atualização de 2018 do Programa nacional de vacinação da Austrália. ${ }^{11}$

Os critérios utilizados para inclusão dos artigos consistiram em: população-alvo constituída por rapazes em idade pediátrica (0 aos 18 anos); intervenção - vacinação contra o HPV no género masculino; em comparação com a não vacinação; e Outcome relacionado com prevenção de patologia oncológica ou pré-maligna por HPV.

Foram utilizados, como critérios de exclusão, estudos não randomizados, artigos duplicados, artigos de opinião, artigos de revisão clássica de tema e artigos discordantes do objetivo da revisão.

Para avaliar o nível de evidência (NE) e a força de recomendação foi utilizada a escala Strengh of Recomendation Taxonomy (SORT), da American Academy of Family Physicians.

\section{RESULTADOS}

Da pesquisa efetuada resultou um total de 532 artigos, dos quais 478 foram excluídos pela leitura do títu- lo e do resumo e 40 foram excluídos após leitura integral; foram incluídos nesta revisão três NOC e 11 estudos originais. Em agosto de 2018 foram incluídas versões mais recentes das três NOC atualizadas.9-11

A Figura 1 representa o fluxograma de seleção dos estudos. O Quadro I resume a descrição das NOC que recomendam a vacinação contra o HPV no género masculino em idade pediátrica. ${ }^{9-11}$ No Quadro II encontram-se os restantes estudos incluídos nesta revisão., ${ }^{6,14-23}$

Nos Estados Unidos, as vacinas quadrivalente e nonavalente contra o HPV estão licenciadas para uso em mulheres e homens com idade entre os 9 e 26 anos. A bivalente está licenciada para uso em mulheres com idade entre os 9 e 25 anos. A partir do final de 2016 apenas a nonavalente está a ser distribuída. O CDC adotou as recomendações da ACIP, que recomenda a vacinação aos 11-12 anos. A vacinação pode ser iniciada aos nove anos. Também recomendam a vacinação de mulheres até aos 26 anos e homens até aos 21 anos que não foram adequadamente vacinados. Para aqueles que iniciaram a vacinação antes dos 15 anos, o esquema recomendado é o de duas doses (a $2^{\text {a }}$ dose deve ser administrada seis a 12 meses após a $1^{\text {a }}$ dose). Para os que iniciaram a vacinação a partir dos 15 anos já são recomendadas três doses (a $2^{\text {a }}$ dose deve ser administrada um a dois meses após a $1^{\text {a }}$ dose e a $3^{\text {a }}$ dose deve ser administrada seis meses após a $1^{\text {a }}$ dose). Os homens que praticam sexo com outros homens têm alto risco de infeção pelo HPV, pelo que também está recomendada a sua vacinação de rotina até aos 26 anos de idade (para aqueles que não foram adequadamente vacinados). ${ }^{9}$

No Canadá, o NACI recomenda as vacinas quadrivalente e nonavalente em todos os homens dos nove aos 26 anos, uma vez que parece diminuir a incidência de infeção, neoplasia anal intraepitelial e lesões genitais externas nos jovens na faixa etária dos 16 e os 26 anos. A bivalente não está indicada. Está recomendada a vacinação do género masculino dos nove aos 14 anos com duas ou três doses com a vacina quadrivalente ou com três doses da vacina nonavalente. Naqueles com idade superior ou igual a 15 anos estão recomendadas as três doses da vacina quadrivalente ou nonavalente. ${ }^{10}$

Na Austrália, a Royal Australian College of General Practitioners (RACGP) inclui a vacina contra o HPV no programa nacional de vacinação e recomenda-a em 


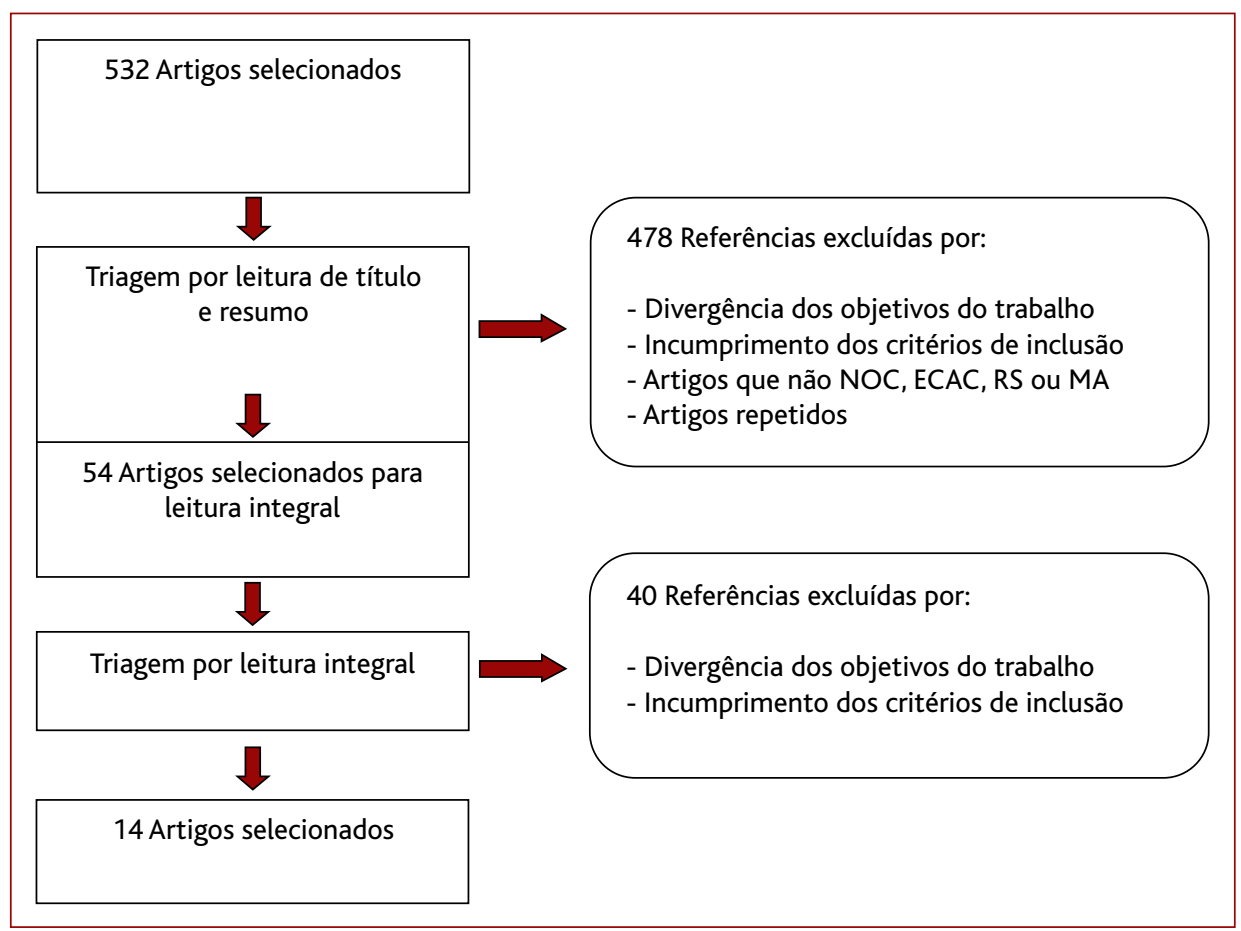

Figura 1. Fluxograma de seleção dos artigos.

Legenda: $\mathrm{NOC}=$ Norma de orientação clínica; $\mathrm{ECAC}=$ Ensaios clínicos aleatorizados; $\mathrm{RS}=$ Revisão sistemática; MA = Meta-análise.

ambos os géneros, entre os 12 e os 13 anos, sendo recomendada a vacina nonavalente (duas doses com um intervalo de seis a 12 meses). Todos os indivíduos (homens e mulheres) que iniciam a vacinação entre os nove e os 14 anos de idade, exceto indivíduos imunocom- prometidos, devem receber duas doses da vacina nonavalente com intervalo entre seis e 12 meses (0,6-12 meses). ${ }^{11}$

Na Europa não há NOC que recomende a vacina contra o HPV no género masculino. Contudo, a $\mathrm{Eu}$ ropean Centre for Disease Prevention and Control (ECDC) faz referência ao facto de apenas a vacina quadrivalente ter sido avaliada quanto à eficácia e relatada como sendo eficaz na prevenção de infeções persistentes e morbilidade relacionada com o HPV em rapazes. Em dois estudos sobre a imunogenicidade da vacina contra o HPV-16/18, a resposta imunológica foi substancialmente maior em jovens do género masculino, em comparação com as mulheres de grupos etários semelhantes. Os níveis de anticorpos após a vacinação para as vacinas de tipo HPV foram até três vezes maiores em homens que em mulheres. As vacinas provocaram níveis de anticorpos mais elevados em gru-

\section{QUADRO I. Normas de orientação clínica (NOC)}

\begin{tabular}{|c|c|c|}
\hline Referência & Recomendações & Força de recomendação \\
\hline $\begin{array}{l}\text { Advisory Committee on } \\
\text { Immunization Practices } \\
\text { [ACIP] (2016) }\end{array}$ & $\begin{array}{l}\text { Vacinação de rotina aos } 11 \text { ou } 12 \text { anos (pode ser iniciada aos } 9 \text { anos). } \\
\text { Vacinação entre os } 13 \text { e os } 21 \text { anos para os que não foram } \\
\text { previamente vacinados ou que não completaram as duas doses. }\end{array}$ & $A$ \\
\hline $\begin{array}{l}\text { National Advisory } \\
\text { Committee on } \\
\text { Immunization [NACl] } \\
(2017)\end{array}$ & $\begin{array}{l}\text { Vacinação entre os } 9 \text { e os } 26 \text { anos. } \\
\text { Vacinação género masculino 9-14 anos com duas ou três doses com a } \\
\text { vacina quadrivalente ou com três } 3 \text { doses da vacina nonavalente. } \\
\text { Vacinação do género masculino com idade superior ou igual a } 15 \text { anos } \\
\text { com três doses da vacina quadrivalente ou nonavalente. }\end{array}$ & B \\
\hline $\begin{array}{l}\text { Royal Australian College } \\
\text { of General Practitioners } \\
\text { (2018) }\end{array}$ & $\begin{array}{l}\text { Vacinação de rotina dos } 9 \text { aos } 14 \text { anos de idade, com duas doses da } \\
\text { vacina nonavalente. }\end{array}$ & $A$ \\
\hline
\end{tabular}




\section{QUADRO II. Resumo dos estudos incluídos no trabalho}

\begin{tabular}{|c|c|c|c|c|}
\hline Referência & Tipo & População/Intervenção & Resultados & NE \\
\hline $\begin{array}{l}\text { Bogaards, et al. } \\
\text { (2015) }\end{array}$ & EOR & $\begin{array}{l}\text { Adolescentes do género masculino de } 12 \text { anos. } \\
\text { Avaliação do efeito da vacinação contra o HPV } \\
\text { bivalente quanto ao ganho de anos de vida } \\
\text { ajustados por qualidade e na redução do } \\
\text { cancro (orofaringe, peniano, anal) nos homens. }\end{array}$ & $\begin{array}{l}\text { A vacinação apenas dos rapazes leva a um } \\
\text { ganho de } 14,6 \text { anos de vida ajustados por } \\
\text { qualidade (IC } 95 \% ; 11,9-17,7) \text {. Existe benefício } \\
\text { na introdução de vacina bivalente no PNV } \\
\text { para rapazes. }\end{array}$ & 2 \\
\hline $\begin{array}{l}\text { Elfstrom, et al. } \\
(2015)\end{array}$ & EOR & $\begin{array}{l}\text { Comparar a prevenção de infeção por HPV } \\
\text { com vacinação bivalente no género feminino } \\
\text { dos } 10-12 \text { anos em relação a extensão única } \\
\text { da vacinação a raparigas dos } 22-26 \text { anos e a } \\
\text { rapazes em idade escolar, em relação a } \\
\text { extensão a homens entre } 25-30 \text { anos. }\end{array}$ & $\begin{array}{l}\text { Vacinação contra HPV mista ou com extensão } \\
\text { ao género masculino em idade adulta acelera } \\
\text { a redução da infeção por HPV e cancro cervical } \\
\text { e aumenta o impacto da vacinação. }\end{array}$ & 2 \\
\hline $\begin{array}{l}\text { Ferris, et al. } \\
(2014)\end{array}$ & ECR & $\begin{array}{l}N=1.781 \text { rapazes e raparigas entre os 9-15 } \\
\text { anos. } \\
\text { Vacinação contra HPV quadrivalente para } \\
\text { avaliação serológica do nível de anticorpos de } \\
\text { modo a verificar a imunogenicidade ao longo } \\
\text { do tempo e avaliação da segurança e eficácia } \\
\text { da vacina contra a infeção persistente ou } \\
\text { doença. }\end{array}$ & $\begin{array}{l}\text { Vacina quadrivalente apresenta duração de } \\
\text { nível de anticorpos e eficácia clínica por oito } \\
\text { anos. } \\
\text { Vacina é mais eficaz se administrada antes de } \\
\text { aquisição do vírus. }\end{array}$ & 2 \\
\hline $\begin{array}{l}\text { Lehtinena, et al. } \\
\text { (2015) }\end{array}$ & ECR & $\begin{array}{l}N=32.175,20.514 \text { raparigas e } 11.661 \text { rapazes. } \\
\text { Avaliar o efeito da vacinação com HPV } \\
\text { bivalente em raparigas comparativamente a } \\
\text { rapazes e raparigas nascidos entre } 1992-1995, \\
\text { nos anos escolares } 2007-2008 \text { e } 2008-2009 \text {. }\end{array}$ & $\begin{array}{l}\text { A vacinação mista aumenta em } 31 \% \text { a } \\
\text { imunidade de grupo. }\end{array}$ & 2 \\
\hline $\begin{array}{l}\text { Marcelon, et al. } \\
\text { (2015) }\end{array}$ & EOR & $\begin{array}{l}\text { Rapazes entre os 9-14 anos. } \\
\text { Quantificar o risco-benefício da vacinação } \\
\text { com HPV quadrivalente em rapazes para } \\
\text { prevenção do cancro anal, em comparação } \\
\text { com a não vacinação. }\end{array}$ & $\begin{array}{l}\text { A vacinação com HPV quadrivalente em } \\
\text { rapazes parece ter um risco-benefício favorável } \\
\text { para prevenção do cancro anal. O grupo de } \\
\text { rapazes mais jovens apresenta uma resposta } \\
\text { imunitária superior à dos adultos. Outro } \\
\text { benefício é a diminuição de condilomas } \\
\text { acuminados. }\end{array}$ & 2 \\
\hline $\begin{array}{l}\text { Korostil, et al. } \\
\text { (2013) }\end{array}$ & EOR & $\begin{array}{l}\text { Predição do impacto da vacinação em crianças } \\
\text { do género masculino na incidência de lesões } \\
\text { genitais associadas ao HPV, através de um } \\
\text { modelo matemático de transmissão do HPV } \\
\text { na população heterossexual australiana. }\end{array}$ & $\begin{array}{l}\text { Introdução da vacina no género masculino } \\
\text { está associada a uma maior redução da } \\
\text { incidência de lesões genitais na população } \\
\text { heterossexual quando comparada com a } \\
\text { vacinação isolada do género feminino. }\end{array}$ & 2 \\
\hline $\begin{array}{l}\text { Li, et al. } \\
\text { (2012) }\end{array}$ & ECR & $\begin{array}{l}\mathrm{N}=600 \text { (média de idades: } 24,6 \text { ), } 100 \text { do género } \\
\text { masculino e } 500 \text { do género feminino, da } \\
\text { população chinesa, entre os } 9 \text { e os } 45 \text { anos. } \\
\text { Avaliar a segurança e imunogenicidade da } \\
\text { vacina do HPV quadrivalente, após } \\
\text { administração ao dia 1, mês } 2 \text { e mês } 6 \text {. }\end{array}$ & $\begin{array}{l}\text { Os jovens de género feminino e masculino } \\
\text { (9-15 anos) apresentam maior imunogenicidade } \\
\text { (1,4-2,8 vezes superior) do que as mulheres } \\
\text { adultas ( } 16-26 \text { anos). A vacinação em crianças } \\
\text { do género feminino e masculino beneficia a } \\
\text { redução de cancro genital e cervical, lesões } \\
\text { pré-cancerígenas e genitais. }\end{array}$ & 2 \\
\hline
\end{tabular}




\begin{tabular}{|c|c|c|c|c|}
\hline Referência & Tipo & População/Intervenção & Resultados & NE \\
\hline $\begin{array}{l}\text { Bauer, et al. } \\
\text { (2012) }\end{array}$ & EOR & $\begin{array}{l}N=1.754 .000 \text { mulheres e } 258.000 \text { homens. } \\
\text { Avaliar a redução de lesões genitais benignas } \\
\text { após a introdução da vacina HPV. }\end{array}$ & $\begin{array}{l}\text { O diagnóstico de verrugas diminuiu } 34,8 \% \text { em } \\
\text { mulheres com < } 21 \text { anos e } 10 \% \text { com } 21-25 \\
\text { anos; diminuiu } 18,6 \% \text { em homens com < } 21 \\
\text { anos e } 11,2 \% \text { com } 21-25 \text { anos; aumentou } \\
10,1 \% \text { em mulheres com } 26-30 \text { anos e } 9,4 \% \\
\text { com > } 30 \text { anos; e aumentou } 9 \% \text { em homens } \\
\text { entre os } 26-30 \text { anos. }\end{array}$ & 3 \\
\hline $\begin{array}{l}\text { Chesson, et al. } \\
\text { (2011) }\end{array}$ & EOR & $\begin{array}{l}\text { Estimativa do custo-eficiência da inclusão da } \\
\text { vacina do HPV quadrivalente em crianças do } \\
\text { género masculino com } 12 \text { anos, com base na } \\
\text { incidência, custo por caso e impacto na } \\
\text { qualidade de vida durante um período de } \\
\text { tempo de } 100 \text { anos de vacinação. }\end{array}$ & $\begin{array}{l}\text { A vacinação nas crianças do género masculino } \\
\text { leva a maior redução a longo prazo do cancro } \\
\text { cervical associado ao HPV, comparativamente } \\
\text { à vacinação isolada do género feminino. }\end{array}$ & B \\
\hline $\begin{array}{l}\text { Giuliano, et al. } \\
\text { (2011) }\end{array}$ & ECR & $\begin{array}{l}\text { N=4.065 rapazes e homens saudáveis (16-26 } \\
\text { anos) de } 18 \text { países. } \\
\text { Avaliar eficácia da vacina HPV quadrivalente } \\
\text { na redução da incidência de infeção anogenital } \\
\text { e lesões genitais externas relacionadas com o } \\
\text { HPV. }\end{array}$ & $\begin{array}{l}\text { A vacina quadrivalente previne a infeção } \\
\text { anogenital e o desenvolvimento de lesões } \\
\text { genitais externas relacionadas com o HPV em } \\
\text { homens entre os } 16 \text { a } 26 \text { anos de idade. }\end{array}$ & 2 \\
\hline $\begin{array}{l}\text { Block, et al. } \\
(2006)\end{array}$ & ECR & $\begin{array}{l}\mathrm{N}=1.529 \text {, entre } 10 \text { e } 23 \text { anos. } \\
\text { Demonstrar a não inferioridade da } \\
\text { imunogenicidade da vacina quadrivalente } \\
\text { contra o HPV em jovens, meninas e meninos, } \\
\text { comparativamente com mulheres adultas. }\end{array}$ & $\begin{array}{l}\text { A imunização de rapazes e raparigas dos } 10 \\
\text { aos } 15 \text { anos com a vacina quadrivalente contra } \\
\text { o HPV resultou numa resposta imunitária } \\
\text { robusta. Estas respostas de anticorpos } \\
\text { neutralizantes foram estatisticamente não } \\
\text { inferiores às observadas na população dos } 16 \\
\text { aos } 23 \text { anos de idade. }\end{array}$ & 2 \\
\hline
\end{tabular}

Legenda: $\mathrm{ECR}=$ Estudo controlado e randomizado; EOR = Estudo observacional retrospetivo; HPV = Papilomavírus humano.

pos etários mais jovens (10-14 anos em comparação a 15-18 anos) em ambos os géneros. ${ }^{12-13}$

A European Guidelines for Quality Assurance in Cervical Cancer Screening faz referência à vacinação contra o HPV no género masculino, citando estudos que demonstram a eficácia da vacina quadrivalente em homens contra a infeção persistente com HPV 16 e 18, verrugas genitais e lesões anais intraepiteliais. Referem ainda a importância da imunidade de grupo, isto é, a vacinação no género masculino não só tem benefícios diretos para a pessoa vacinada, mas também diminui a circulação do HPV e o risco de infeções por HPV em mulheres e homens não vacinados. ${ }^{13}$

Bogaards e colaboradores (2015) realizaram um es- tudo retrospetivo, com base num modelo bayseano, em que se utiliza uma população de adolescentes de 12 anos do género masculino, na Holanda. O objetivo foi avaliar o efeito da vacina bivalente nos rapazes, através da sua introdução no PNV, calculando o ganho de anos de vida ajustados por qualidade e a redução do cancro (orofaringe, peniano, anal) nos homens. A vacinação apenas de rapazes levou a um ganho de 14,6 anos de vida ajustados por qualidade (IC95\%; 11,9-17,7), sendo que seriam necessários vacinar 466 rapazes para prevenir um caso de cancro (IC95\%; 405-542). O ganho é superior nos homossexuais em comparação com os heterossexuais. Quanto maior a taxa de vacinação do género feminino associado ao masculino, menor é o ga- 
nho de anos de vida ajustados por qualidade e é necessário vacinar um maior número de rapazes para prevenir um caso de cancro. Assim, concluem que existe benefício na introdução de vacina bivalente para rapazes no PNV (nível de evidência 2). ${ }^{14}$

Elfstrom e colaboradores (2015) efetuaram um estudo retrospetivo para comparar a prevenção de infeção por HPV com a vacina bivalente no PNV da Suécia. O objetivo foi avaliar a eficácia no género feminino dos 10 -12 anos comparativamente à extensão única da vacinação a raparigas dos 22-26 anos e com rapazes em idade escolar vacinados/não vacinados e extensão a homens entre 25-30 anos. Verificou-se uma redução da prevalência de HPV no programa com extensão a raparigas $(49,4 \%)$ e com extensão a rapazes $(55,6 \%)$. A vacinação mista contra o HPV ou com extensão ao género masculino, em idade adulta, acelerou a redução da infeção por HPV e cancro cervical. Assim, concluem que a vacinação de rapazes de 11 anos protege contra a diminuição de eficácia por diminuição da cobertura vacinal temporária nas raparigas (nível de evidência 2). ${ }^{15}$

Lehtinena e colaboradores (2015) realizaram um estudo randomizado controlado, com uma população de 32.175 indivíduos, 20.514 raparigas e 11.661 rapazes, para avaliar o efeito da vacina bivalente apenas em raparigas em comparação com a vacinação de rapazes e raparigas nascidos entre 1992-1995 e nos anos escolares 2007-2008 e 2008-2009, na Finlândia. Verificaram que a vacinação mista aumenta em $31 \%$ a imunidade de grupo em comparação com a vacinação apenas de raparigas (nível de evidência 2). ${ }^{16}$

Marcelon e colaboradores (2015) concretizaram um estudo observacional retrospetivo que visa quantificar o risco-benefício da vacina quadrivalente em rapazes entre os 9-14 anos para prevenção do cancro anal, em comparação com a não vacinação. A vacina quadrivalente pareceu ter um risco-benefício favorável para prevenção do cancro anal. O grupo de rapazes mais jovens apresentou uma resposta imunitária superior aos adultos. Outro benefício foi a diminuição de condilomas acuminados (nível de evidência 2). ${ }^{17}$

Ferris e colaboradores (2014) realizaram um estudo randomizado controlado com 1.781 indivíduos rapazes e raparigas entre os 9-15 anos, sem início da atividade sexual. O grupo $1(N=1.179)$ fez a vacina quadrivalente no dia 1 , ao $2^{\circ}$ mês e ao $6^{\circ}$ mês de estudo. O grupo $2(N=$ 482) recebeu a vacina após o mês 30 . Ambos os grupos foram seguidos por 96 meses. O objetivo foi a avaliação serológica do nível de anticorpos nos grupos ao longo do tempo e avaliação da segurança e eficácia da vacina contra a infeção persistente ou doença. A vacina quadrivalente apresenta duração de nível de anticorpos e eficácia clínica por oito anos. Aos 96 meses, o nível de IgG foi de 94,3\% para o HPV6, 89,4\% para o HPV11, 99,5\% para o HPV16 e 88,8\% para o HPV18. A vacina foi mais eficaz no Grupo 1, não havendo doença ou infeção por HPV por mais do que um ano. Assim, concluíram que a vacina é mais eficaz se administrada antes de aquisição do vírus, não tendo sido encontrados efeitos adversos significativos (nível de evidência 2). ${ }^{6}$

Korostil e colaboradores (2013) realizaram um estudo retrospetivo e transversal, onde foi utilizado um modelo matemático dinâmico da transmissão do HPV-6/11, na população australiana heterossexual, para predizer a alteração na incidência de lesões genitais com a vacinação, desde 2007. Assumiram uma taxa de cobertura de $80 \%$ da atual vacinação feminina e consideraram o início da vacinação masculina em 2013. Quanto aos resultados, a previsão do modelo e os dados relatados até 2013 mostraram uma diminuição relativa a curto prazo da incidência de lesões genitais; desde 2013, o modelo prediz uma redução relativa de aproximadamente $70 \%$ em mulheres e $65 \%$ em homens, após seis anos de vacinação. O efeito da vacinação masculina, após 2013, será substancial por volta de 2020 e aumentará consistentemente até 2030. A redução relativa da incidência de lesões genitais em mulheres será de $80 \%$ em 2021, o que é 7\% adicional à redução comparada só com a vacinação feminina. Da mesma forma, a redução nos homens alcançará os $75 \%$, $12 \%$ superior à vacinação exclusivamente feminina. Baseado nas previsões do modelo, os autores esperam que, em 2030, a redução relativa nas mulheres exceda os $90 \%$ e se aproxime de $90 \%$ nos homens, com a vacinação de ambos os géneros (sem a vacinação masculina, a redução seria menor que $80 \%$ nas mulheres e perto de $65 \%$ nos homens). ${ }^{18}$

Li e colaboradores (2012) fizeram um estudo aleatorizado, duplamente cego e controlado com placebo em crianças e adultos chineses saudáveis dos géneros masculino e feminino, com o objetivo de determinar a efi- 
cácia e a imunogenicidade da vacina quadrivalente do HPV. O estudo foi realizado entre julho e agosto de 2008 e foram aleatorizadas 100 crianças do género masculino com idades entre os nove e os 15 anos e 500 mulheres com idades entre os nove e os 45 anos, na razão 1:1, para receber a vacina quadrivalente Gardasil ${ }^{\circledR}$ ou placebo adjuvante. $O$ ensaio excluiu mulheres pós-pubertárias que estiveram grávidas ou que tiveram história de patologia do colo uterino. Dos 600 participantes, 588 (98\%) receberam as três doses como planeado, foram a todas as consultas e completaram o estudo ao mês 7 . Relativamente à imunogenicidade, a taxa de seroconversão para o HPV6, 11, 16 e 18 ao mês 7 foram de 96,7\%, 99,3\%, 99,3\% e 99\%, respetivamente (a resposta a cada tipo de HPV foi considerada aceitável). As crianças, quer do género masculino quer do feminino, tiveram uma resposta imunitária 1.4-2.8 vezes superior às idades mais velhas. Quanto à segurança, a vacina foi bem tolerada no geral, não havendo diferenças significativas nos efeitos adversos sistémicos entre a vacina e o placebo. $^{19}$

Bauer e colaboradores (2012) realizaram um estudo ecológico, entre 2007 e 2010, com utentes do Programa de Tratamento e Acesso de Cuidados do Planeamento Familiar da Califórnia para avaliar a redução de lesões genitais benignas após a introdução da vacina HPV. Não obtiveram dados sobre o estado vacinal. Foram avaliados 1.754 .000 casos de mulheres e 258.000 de homens. O diagnóstico de verrugas diminuiu 34,8\% em mulheres com menos de 21 anos e $10 \%$ com 21-25 anos; diminuiu 18,6\% em homens com menos de 21 anos e $11,2 \%$ com $21-25$ anos; aumentou 10,1\% em mulheres entre os 26 e 30 anos e 9,4\% acima dos 30 anos; e aumentou 9\% em homens entre os 26-30 anos (não houve alteração nos homens acima dos 30 anos)..$^{20}$

Chesson e colaboradores (2011) realizaram um estudo retrospetivo e transversal em que estimaram o custo-eficiência da inclusão da vacina do HPV quadrivalente em crianças do género masculino, com 12 anos, dos Estados Unidos, com base na incidência, custo por caso e impacto na qualidade de vida, durante um período de tempo de 100 anos de vacinação. Utilizaram um modelo simplificado da transmissão do HPV e dirigiram três questões: o custo-eficiência da vacinação no género feminino, entre os 12-26 anos, comparado com a não vacinação; o custo-eficiência de adicionar a vacinação no género masculino para as idades entre os 12 e 26 anos; e o custo-eficiência de abranger a vacinação nos rapazes de 12 anos, comparado com a estratégia de aumentar a cobertura da vacina nas raparigas de 12 anos. A inclusão da vacinação nas crianças do género masculino reduziu a longo prazo o cancro cervical associado ao HPV em 81,3\%, 64,7\% e 97,4\%, com taxas de cobertura de $30 \%$, $20 \%$ e $75 \%$, respetivamente (em comparação com a redução de $71,1 \%, 55,2 \%$ e $94,6 \%$ na vacinação só de crianças do género feminino). No entanto, demonstraram que o custo-eficiência da vacinação em crianças do género masculino depende da cobertura no género feminino, sendo mais custo-eficiente quando a taxa de cobertura da vacinação no género feminino é baixa. Aumentar a taxa de cobertura da vacinação no género feminino pode ser mais custo-eficiente que a vacinação no género masculino. ${ }^{21}$

Giuliano e colaboradores (2011) efetuaram um estudo randomizado, duplamente cego, controlado com placebo, que incluiu 4.065 rapazes saudáveis de 18 países, entre os 16 e os 26 anos de idade. Foram excluídos os indivíduos com história prévia ou atual de lesões clinicamente sugestivas de infeção sexualmente transmissível. Os sujeitos do estudo foram divididos por dois grupos, 2.032 para a vacina e 2.033 para placebo, ambos vacinados no dia 1, mês 2 e mês 6 . O tempo médio de seguimento após a primeira administração de vacina ou placebo foi de 2,9 anos. O objetivo primário foi avaliar a eficácia da vacina quadrivalente na redução da incidência de lesões genitais externas relacionadas aos serotipos 6, 11, 16 ou 18. Um total de 175 indivíduos não retornaram para o seguimento após receberem uma dose de vacina ou placebo. As análises de eficácia foram realizadas através de protocolos para duas populações distintas: indivíduos que receberam as três vacinas e foram negativos para os tipos relevantes de HPV no início do estudo; indivíduos que receberam vacina ou placebo, independentemente do estado basal do HPV, onde havia intenção de tratar. A maioria dos indivíduos que foram inicialmente negativos para os quatro serotipos envolvidos na vacina, tiveram seroconversão para todos os serotipos um mês após a terceira dose de vacina $(97,5 \%)$. Na população de intenção de tratamento, a eficácia contra lesões relacionadas com estes serotipos foi de 65,5\% (IC95\%; 45,8-78,6) e a eficácia na prevenção de qualquer lesão genital, inde- 
pendentemente do tipo HPV, foi de 60,2\% (IC95\%; 40,8-73,8). A vacina foi também eficaz contra a infeção persistente pelos serotipos da vacina e contra a deteção de DNA para estes tipos de HPV. Na população de indivíduos seronegativos, no início do estudo, a vacina reduziu a incidência de lesões genitais externas relacionadas aos serotipos 6, 11, 16 ou 18 em 90,4\% (IC95\%; 69,2$-98,1)$ e a eficácia contra condilomas acuminados foi de 89,4\% (IC95\%; 65,5-97,9). Não foram encontrados efeitos adversos significativos. Embora as estimativas de eficácia neste estudo sejam numericamente mais baixas do que as de meninas e mulheres em outros estudos, os intervalos de confiança sobrepõem-se, sugerindo que a eficácia da vacina pode ser semelhante para os dois géneros. ${ }^{22}$

Block e colaboradores (2006) realizaram um ensaio clínico, multicêntrico, em diversos países, de não inferioridade da imunogenicidade da vacina quadrivalente em jovens, meninas e meninos, comparativamente com mulheres adultas. Foram incluídas 506 meninas e 510 meninos dos 10 aos 15 anos, saudáveis, sem atividade sexual antes e durante o estudo, que foram comparados com 513 mulheres entre os 16 e os 23 anos, saudáveis, sem história de alterações no papanicolau, cervicite purulenta, verrugas genitais e não mais de quatro parceiros sexuais. Todos os participantes foram vacinados no dia 1 , mês 2 e mês 6 . O tempo médio de seguimento após a primeira administração de vacina foi de um ano. A tolerabilidade da vacina também foi avaliada. As serologias foram pedidas no dia 1 e nos meses 3 e 7, sendo as taxas de seroconversão no sétimo mês de $99 \%$ para todos os quatro tipos de HPV em cada grupo e os títulos médios nas meninas ou meninos 1,7 a 2,7 vezes maiores. As respostas imunogénicas não inferiores para os quatro serotipos de HPV presentes na vacina quadrivalente permitem a transposição de dados de eficácia que foram gerados em mulheres jovens para as raparigas. Os resultados nos meninos apoiam a implementação de programas de vacinação também para o género masculino. ${ }^{23}$

\section{CONCLUSÕES}

A vacinação contra o HPV no sexo masculino tem suscitado debates éticos e morais, sendo ainda contestada por diversos motivos: as vacinas comercializadas em Portugal não representam uma parte significativa dos mais de 200 serotipos oncogénicos conhecidos, apesar de serem eficazes contra os mais prevalentes; os restantes serotipos não abrangidos pelas vacinas podem acumular mutações e adquirirem maior potencial oncogénico e/ou infecioso.

O principal argumento contra a vacinação masculina é baseado nos custos associados que foram estimados por modelos de custo-benefício. Entre os diversos estudos realizados, a maioria concorda que a vacinação de homens e mulheres é menos custo-efetiva que a vacinação exclusiva das mulheres. Estudos de custo-efetividade da vacinação contra o HPV nos rapazes têm demonstrado que esta está muito associada à cobertura vacinal das raparigas. A cobertura vacinal das raparigas contra o HPV é suficientemente alta. Tem-se discutido então se a implementação da vacinação nos rapazes resultará em benefícios adicionais que justifiquem os custos da intervenção. É, no entanto, considerável a variabilidade nos valores utilizados nos parâmetros necessários à construção destes modelos de custo-benefício.

O HPV tem uma elevada incidência, em ambos os géneros, de lesões benignas e neoplasias malignas. A forma de reduzir individualmente o risco de doença é através da vacinação, para além da proteção indireta, com método de barreira.

Ainda que de forma lenta e contrariada pela dívida no Sistema Nacional de Saúde, a vacina é cada vez mais acessível e as normas lançadas pela Direção-Geral da Saúde caminham para a sua introdução no Programa Nacional de Vacinação também no género masculino.

Segundo a revisão dos estudos mencionados e o parecer das Sociedades Portuguesas de Pediatria e de Infeciologia Pediátrica, a vacinação no género masculino é recomendada, pois a doença (condilomas e neoplasias do pénis, ânus, cabeça e pescoço) é clínica e epidemiologicamente relevante; não existe rastreio implementado; e é importante pensar nos homens que praticam sexo com outros homens e que não ficam protegidos pela vacinação exclusiva no género feminino. A vacina tem um bom perfil de segurança e tudo indica que tem o potencial de reduzir uma proporção significativa de doenças que afetam o homem, além de impulsionar a proteção no sexo feminino.

Em suma, a evidência atual disponível indica que a vacinação contra o HPV está indicada no género mas- 
culino em idade pediátrica (força de recomendação A), apresentando uma relação custo-benefício efetiva. A vacinação mista é mais eficaz do que a vacinação isolada do género feminino. Existe maior eficácia se administrada antes de iniciar uma vida sexual ativa. A vacinação nesta faixa etária reduz a incidência de infeção pelo HPV e, consequentemente, o número de lesões malignas e pré-malignas. Esta redução é mais preponderante em países com menor taxa de vacinação no género feminino.

Será, assim, importante as autoridades de saúde partirem para uma estratégia de vacinação do HPV mais abrangente.

\section{AGRADECIMENTOS E ESCLARECIMENTOS}

Este trabalho foi realizado no âmbito do Internato Complementar em Medicina Geral e Familiar.

\section{REFERÊNCIAS BIBLIOGRÁFICAS}

1. Boiron L, Joura E, Largeron N, Prager B, Uhart M. Estimating the costeffectiveness profile of a universal vaccination programme with a ninevalent HPV vaccine in Austria. BMC Infect Dis. 2016;16:153.

2. Giuliano AR, Palefsky JM, Goldstone S, Moreira ED Jr, Penny ME, Aranda $C$, et al. Efficacy of quadrivalent HPV vaccine against HPV Infection and disease in males. N Engl J Med. 2011;364(5):401-11.

3. Burger EA, Sy S, Nygård M, Kristiansen IS, Kim JJ. Prevention of HPVrelated cancers in Norway: cost-effectiveness of expanding the HPV vaccination program to include pre-adolescent boys. PLoS One. 2014;9(3):e89974.

4. Leça A, Sarmento AM, Freitas G, Marques JG, Marques LH, Santos LA, et al. Plano nacional de vacinação 2017. Lisboa: Direção-Geral da Saúde; 2016.

5. Graham DM, Isaranuwatchai W, Habbous S, de Oliveira C, Liu G, Siu LL, et al. A cost-effectiveness analysis of human papillomavirus vaccination of boys for the prevention of oropharyngeal cancer. Cancer. 2015;121(11):1785-92.

6. Ferris D, Samakoses R, Block S, Lazcano-Ponce E, Restrepo J, Reisinger $K$ et al. Ferris D, Samakoses R, Block SL, Lazcano-Ponce E, Restrepo JA, Reisinger KS, et al. Long-term study of a quadrivalent human papillomavirus vaccine. Pediatrics. 2014;134(3):e657-65.

7. Garnock-Jones KP, Giuliano AR. Quadrivalent human papillomavirus (HPV) types 6, 11, 16, 18 vaccine: for the prevention of genital warts in males. Drugs. 2011;71(5):591-602.

8. Sharma M, Sy S, Kim JJ. The value of male human papillomavirus vaccination in preventing cervical cancer and genital warts in a low-resource setting. BJOG. 2016;123(6):917-26.

9. Meites E, Kempe A, Markowitz LE. Use of a 2-dose schedule for human papillomavirus vaccination: updated recommendations of the Advisory Committee on Immunization Practices. MMWR Morb Mortal Wkly Rep. 2016;65(49):1405-8.

10. National Advisory Committee on Immunization. Updated recommen- dations on human papillomavirus (HPV) vaccines: 9-valent HPV vaccine 2-dose immunization schedule and the use of HPV vaccines in immunocompromised populations. Ottawa: NACI; 2017. ISBN 9780660072906

11. Australian Technical Advisory Group on Immunisation. The Australian immunisation handbook [website]. 10th ed. Canberra: Australian Government Department of Health; updated 2018. Available from: https://immunisationhandbook.health.gov.au/

12. Simone B, Carrillo-Santisteve P, Lopalco P. Introduction of HPV vaccines in European Union countries: an update. Stockholm: European Centre for Disease Prevention and Control; 2012. ISBN 9789291933778

13. Arbyn M, Anttila A, Jordan J, Ronco G, Schenck U, Segnan N, et al. European guidelines for quality assurance in cervical cancer screening: second edition - summary document. Ann Oncol. 2010;21(3):448-58.

14. Bogaards JA, Wallinga J, Brakenhoff RH, Meijer CJ, Berkhof J. Direct benefit of vaccinating boys along with girls against oncogenic human papillomavirus: bayesian evidence synthesis. BMJ. 2015;350:h2016.

15. Elfström KM, Lazzarato F, Franceschi S, Dillner J, Baussano I. Human papillomavirus vaccination of boys and extended catch-up vaccination: effects on the resilience of programs. J Infect Dis. 2016;213(2):199-205.

16. Lehtinen M, Apter D, Baussano I, Eriksson T, Natunen K, Paavonen J, et al. Characteristics of a cluster-randomized phase IV human papillomavirus vaccination effectiveness. Vaccine. 2015;33(10):1284-90.

17. Marcelon L, Verstraeten T, Dominiak-Felden G, Simondon F. Quantitative benefit-risk assessment by MCDA of the quadrivalent HPV vaccine for preventing anal cancer in males. Expert Rev Vaccines. 2016;15(1):139-48.

18. Korostil IA, Ali H, Guy RJ, Donovan B, Law MG, Regan DG. Near elimination of genital warts in Australia predicted with extension of human papillomavirus vaccination to males. Sex Transm Dis. 2013;40(11):8335.

19. Li R, Li Y, Radley D, Liu Y, Huang T, Sings HL, et al. Safety and immunogenicity of a vaccine targeting human papillomavirus types $6,11,16$ and 18: a randomized, double-blind, placebo-controlled trial in Chinese males and females. Vaccine. 2012;30(28):4284-91.

20. Bauer HM, Wright G, Chow J. Evidence of human papillomavirus vaccine effectiveness in reducing genital warts: an analysis of California public family planning administrative claims data, 2007-2010. Am J Public Health. 2012;102(5):833-5.

21. Chesson HW, Ekwueme DU, Saraiya M, Dunne EF, Markowitz LE. The cost-effectiveness of male HPV vaccination in the United States. Vaccine. 2011;29(46):8443-50.

\section{CONFLITO DE INTERESSES}

As autoras declaram não ter quaisquer conflitos de interesse.

\section{ENDEREÇO PARA CORRESPONDÊNCIA}

Vanessa Filipa Guedes Silva

E-mail:vgsilva10@gmail.com

https://orcid.org/0000-0001-7318-5470

Recebido em 21-11-2017

Aceite para publicação em 09-12-2018 


\section{ABSTRACT}

IMMUNISATION AGAINST THE PAPILLOMAVIRUS IN MALES OF PEDIATRIC AGE: WHAT'S THE EVIDENCE?

Objective: To evaluate the efficacy of the vaccination against the human papillomavirus (HPV) in males in pediatric age, in the prevention of malignant or pre-malignant HPV-associated lesions.

Data sources: PubMed, Cochrane Library, DARE, National Guideline Clearinghouse, Canadian Medical Association Practice Guidelines InfoBase, Guidelines Finder (National Electronic Library for Health NHS England).

Methods: Search of articles published between 01/01/2006 and 01/09/2019, using the MeSH terms 'papillomavirus vaccines', 'male', 'children', and 'adolescent'. Male individuals of pediatric age, immunised against HPV for prevention of malignant and pre-malignant HPV-associated lesions were included. The level of evidence was graded according to The American Academy of Family Physicians' Strength of Recommendation Taxonomy Scale (SORT).

Results: In the three clinical guidelines included, male vaccination for HPV in males of pediatric age is recommended with strength of recommendation A. Of the 11 articles included, the majority considers that immunization against HPV in males of pediatric age reduces the incidence of HPV infection, and of malignant and pre-malignant lesions.

Conclusion: Immunisation against HPV is effective in preventing HPV lesions and is therefore recommended in males in pediatric age (strength of recommendation A). The vaccination of individuals of both genders is more effective than female vaccination only.

Keywords: Adolescent; Children; Male; Papillomavirus vaccine. 